\title{
UN ANÁLISIS DE LOS DETERMINANTES \\ DEL PRECIO DE LOS ESCLAVOS \\ HISPANOAMERICANOS EN EL SIGLO XVIII *
}

\author{
CARLOS NEWLAND Y MARIA JESÚS SAN SEGUNDO \\ Universidad Carlos III de Madrid
}

\section{RESUMEN}

El artículo analiza los determinantes del ciclo vital del precio de los esclavos, concentrándose especialmente en el impacto del capital humano, tanto en capacitación como en salud. Se describe el origen de la muestra utilizada - que incluye más de dos mil esclavos hispanoamericanos del siglo XvIII- y se discute su fiabilidad; más adelante se describen sus características en el marco histórico-geográfico. Se pasa luego a una investigación sobre los factores que afectan los precios para una variedad de actividades económicas.

\section{ABSTRACT}

This paper analizes the determinants of the price life cycle of a slave, dealing particularly with the impact of human capital, both with respect to skills and health. The paper details the source of the sample -including more than two thousand Spanish American slaves of the 18th century- and discusses its reliability, moving on to a descriptive analysis in the geographic and historical context. Later on it looks at the factors influencing price in several types of economic activity.

La historia microeconómica de los mercados laborales es un área que ha recibido escasa atención por parte de especialistas en América Latina. Esto en gran medida es consecuencia de la carencia de información fiable sobre carac-

* Agradecemos la ayuda recibida por Guillermo Conconi Borel, Alberto Vaquero Garcia y Joaquin Paredes Diéguez en la elaboración de la base de datos; y la generosa colaboración de los integrantes del equipo de catalogación del fondo jesuita, del Archivo Histórico Nacional de Chile, María Cristina Picón y Jaime Vivanco González. 
terísticas de los trabajadores y sus ingresos, lo que a su vez impide el más simple análisis econométrico. Una muestra de esta deficiencia es la escasez de series de salarios reales (anteriores al siglo $\mathrm{xx}$ ), lo que dificulta evaluar la evolución del nivel de vida y el influjo de otras variables sobre el ingreso per cápita. Destaca a este respecto la brecha existente con los estudios sobre los Estados Unidos; este fenómeno se ve agravado por la riqueza en la variedad de arreglos legales que existió en América Latina, que incluyó la mita, la encomienda, el trabajo bajo contrato (indentured labor) y la esclavitud.

En este trabajo se intenta hacer una aportación para llenar este vacio, investigando algunas características de los precios de esclavos de Hispanoamérica, imitando un análisis ya efectuado para los Estados Unidos, Brasil y el Caribe ${ }^{1}$. Se analiza una muestra de más de dos mil esclavos del siglo xvin, tratando en particular el impacto sobre los precios del capital humano, tanto en capacitación como en salud. En primer lugar se detalla el origen de la muestra y se discute su fiabilidad, pasando luego a su análisis descriptivo en el contexto temporal geográfico. Más adelante se estudian los determinantes de los precios en diversos tipos de actividades económicas.

\section{EL ORIGEN DE LA MUESTRA Y SU FIABILIDAD}

La muestra que analiza este estudio consiste en información sobre 2.347 esclavos para el periodo 1767-1794. De cada individuo se conoce su precio, edad, sexo, origen criollo o africano, capacitación, salud y tipo de unidad económica en que se desempeñaba. Los datos se originan en las tasaciones que se realizaron sobre todas las propiedades jesuitas, luego de que la orden fue expulsada del territorio español en 1767. Los jesuitas estaban compuestos en América de unos 2.200 miembros, y administraban un numeroso conjunto de establecimientos educativos que abarcaban todos los niveles de enseñanza, incluyendo el universitario. Fue en el nivel secundario donde más se destacaron: sus colegios pasaban del centenar y no tenían mayor competencia local. Dado que estos establecimientos no recibian fondos del Estado debieron financiarse por matrículas cobradas a los alumnos; como esta fuente no era suficiente también adquirieron empresas de todo tipo para obtener fondos adicionales. Éstas incluyeron estancias, haciendas, ingenios, viñedos y actividades semiindustriales o artesanales, como la fabricación de tejas, textiles, sombreros, muebles,

1 Kotlikoff (1992), Fogel y Engerman (1974a), (1974b), (1992); Fogel (1989), Fogel, Galantine y Manning (1992), Higman (1976), Marcilio (1978), Moreno Fraginals, Klein y Engerman (1983), Queirós Mattoso, Klein y Engerman (1986). 
plateria y hasta relojes. Los jesuitas se adaptaron a la mano de obra disponible en cada región, lo que incluyó a trabajadores asalariados donde éstos eran abundantes y a esclavos donde este no era el caso ${ }^{2}$.

Los esclavos jesuitas quedaron registrados en los inventarios que el gobierno español mandó realizar a partir de 1767 para poder controlar los bienes que habian sido confiscados. Para confeccionarlos se contrató en cada sitio a especialistas, los que incluyeron a tasadores profesionales, herreros, carpinteros, albañiles y expertos en esclavos, que en casos fueron acompañados por médicos. Estos peritos, a los que se les pagaron sus servicios, actuaban bajo juramento y amenaza de confiscación de sus propiedades si sus estimaciones no eran fidedignas. Por otra parte, debian ser acompañados por testigos, vecinos conocidos, funcionarios, militares o clérigos, que inhibieran posibles falsificaciones. Los inventarios tienen una calidad variable y son más completos y exactos cuando las propiedades se encontraban cerca de centros urbanos de importancia. Inclusive en casos se hicieron con un detalle excesivo -lo que implicaba aumentar su coste - y desde Madrid se ordenó realizarlos más rápidamente y con menor gasto ${ }^{3}$. En este trabajo se descartaron las valuaciones que no contenian información completa para cada individuo.

Una de las ventajas de la muestra es que todos los esclavos fueron individualmente valorados, por lo que existe información para niños de corta edad, quienes en otro tipo de registros aparecen frecuentemente tasados conjuntamente con sus madres; por ser un registro total, se evita también el problema de selección adversa (que los esclavos que aparecieran en el mercado fueran los peores). Pero, ¿̨cuál es la fiabilidad que nos ofrecen estos inventarios? Aunque no podemos tener una garantía de que los tasadores no manipularon los precios en su beneficio personal, dicho manejo sería relativamente visible, pues el valor de un esclavo era conocido, y su variabilidad menor que el valor de la tierra, construcciones y mejoras. Por otra parte, si un comprador no estaba de acuerdo con los precios podia traer sus propios expertos para realizar una nueva evaluación. Pero quizá el incentivo mayor para que las tasaciones se efectuaran correctamente fue que los esclavos se vendieron en remates públicos, lo que haria obvia cualquier divergencia con la realidad. Lamentable-

2 La orden jesuita no se cuestionó de manera sistemática si el uso de esclavos era lícito moralmente, lo que contrasta con su defensa del indigena y la crítica a que se lo sometiera a la servidumbre. En esto eran hijos de su época, pues prácticamente nadie en el mundo criticó la esclavitud hasta los últimos años del siglo xvil. Por otra parte, parecen haber tratado mejor a los esclavos que los propietarios laicos (Chandler, 1981, pp. 180, 206; Riley, 1976, p. 183) y mientras se conocen revueltas de esclavos en sus propiedades (Bauer, 1990, p. 97), en estos casos se revelarían luego de la expulsión de los religiosos, al empeorar su situación (Kapsoli 1975).

3 AHNM Jesuitas, L. 126, № 25. 
mente no se poseen los precios de venta en subasta para la mayoría de los individuos, aunque la información parcial que ha sobrevivido para el Virreinato del Rio de la Plata sugiere que las valoraciones fueron correctas. En la ciudad de Córdoba siete esclavos de la Hacienda de San Ignacio fueron tasados en 960 pesos y vendidos en 987 pesos ${ }^{4}$. En Salta se remataron 55 esclavos en 1771; para la gran mayoria no existió divergencia entre el precio teórico y real; inclusive un grupo de esclavos adquirió su propia libertad, fenómeno que no era raro en la América Hispana 5 . En Tucumán el precio de sólo cinco de los 26 esclavos vendidos no fue el esperado; allí también algunos esclavos compraron su libertad o la de sus hijos ${ }^{6}$. Debe destacarse que si los valores fueron sesgados hacia la baja, las principales conclusiones de este trabajo sólo se verían afectadas si al mismo tiempo se alteró su estructura interna. Finalmente se debe mencionar que en el caso las propiedades jesuitas del Perú, casi todas ellas fueron vendidas con un descuento de $30 \%$ respecto a su valor de tasación, lo que parecería indicar que los esclavos no pueden haber sufrido una subvaluación significativa ${ }^{7}$.

\section{ANÁLISIS DE LA MUESTRA Y SU CONTEXTO}

Hacia fines del siglo xvin habia en el continente americano unos cuatro millones de esclavos. Por su importancia relativa destacaba el Caribe británico y francés, donde vivia un millón que representaba la mayor parte de la población local -en Jamaica eran el 90\%, en Barbados el 80\%-; en Brasil habia millón y medio y su proporción sobre el total de habitantes era menor, del 46\%. En los Estados Unidos rondaban el millón, un 22\% de su población. En la América Española su importancia relativa era mucho menor, pues sus casi 400.000 esclavos sólo eran el 3\% de los habitantes. Por supuesto, su peso no era homogéneo a lo largo de todo el territorio. En el cuadro 1 se detalla su relevancia por regiones. En primer lugar se encuentra Venezuela, con su necesidad de mano de obra para el cultivo del cacao, con 10,2\% de la población esclava. Siguen las Antillas Españolas con 9,1\%, aunque dentro de ellas destacaba Cuba (con 30\%), que estaba importando trabajo a tasas crecientes para su producción azucarera. Muchas regiones donde la mano de obra se utilizaba en la producción aurifera, de azúcar, vino, aguardientes y en actividades

\footnotetext{
4 AHNM Jesuitas, L. 127, N 54.

5 AHNCh Jesuitas, Libro 149.

- AHNCh Jesuitas, Libro 149.

7 Macera (1966), p. 11.
} 
urbanas domésticas y artesanales se encontraban entre un 4 y un $7 \%$; éstas eran Quito, Perú, Nueva Granada, el Río de la Plata y Paraguay. Finalmente los esclavos tendrian escasa importancia en Chile, Alto Perú y Centroamérica (los existentes, muy concentrados en Panamá) y casi nula en Nueva España, en todos los casos por la abundancia y baratura de la mano de obra libre.

\section{CUADRO 1}

Distribución regional de la esclavitud en Hispanoamérica. Total y muestra.

\begin{tabular}{|c|c|c|c|}
\hline Región & $\begin{array}{l}\text { Proporción de la } \\
\text { población total }\end{array}$ & $\begin{array}{l}\text { Proporción del } \\
\text { total de esclavos }\end{array}$ & $\begin{array}{l}\text { Distribución } \\
\text { de la muestra }\end{array}$ \\
\hline Mexico & 0,2 & 2,4 & \\
\hline Centroamérica & 1,1 & 2,5 & 3,6 \\
\hline Caribe $\ldots \ldots . .$. & 9,1 & 20,4 & 5,6 \\
\hline Nueva Granada & 5,8 & 15,8 & \\
\hline Venezuela ...... & 10,2 & 19,2 & 5,1 \\
\hline Quito $\ldots \ldots \ldots \ldots$ & 6,7 & 7,9 & \\
\hline Perú $\ldots . . . . . . .$. & 6,6 & 22,6 & 39,5 \\
\hline Chile .............. & 2,3 & 3,1 & 2,1 \\
\hline$\ldots \ldots \ldots \ldots \ldots \ldots$ & 4,1 & 1,0 & \\
\hline 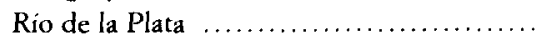 & 4,9 & 5,3 & 44,1 \\
\hline Total & 3,0 & 100,0 & 100,0 \\
\hline
\end{tabular}

FuENTE: Klein (1986), Morales Padrón (1988), Rout (1976).

La distribución geográfica de los individuos que componen la muestra se detalla en el cuadro 1, y sus características principales, en el cuadro 2. Como puede observarse, la misma está sobrerrepresentada para el Río de la Plata y el Perú, aunque también brinda información para Centroamérica, Cuba, Venezuela y Chile. Por área de actividad pueden clasificarse en: 1) Esclavos en establecimientos tipo plantación. Del total general un $36 \%$ se dedicaban al cultivo y procesamiento de la caña de azúcar, un $20 \%$ trabajaba en viñedos en la producción de vino, aguardiente y licores, y un grupo menor se dedicaba al cultivo de cacao (actividad que por su tamaño no se analiza de forma desagregada); 2) Esclavos urbanos - el 12\% - fundamentalmente situados en Montevideo y Buenos Aires, ocupados como artesanos, empleados de los colegios y trabaja- 
dores no calificados; 3) Esclavos de haciendas y estancias, un pequeño grupo dedicado a la ganadería y agricultura, y 4) Esclavos en empresas mixtas -el $22 \%$-, ubicados en establecimientos rurales que combinaban actividades agropecuarias con otras más caracteristicas de zonas urbanas, como producción de tejas, ladrillos o fabricación de muebles. En cuanto a la división por sexos, ésta aparece relativamente equilibrada en todas la actividades, con una ligera preponderancia masculina; en su inmensa mayoría los esclavos formaban parte de unidades familiares constituidas. La excepción es Cuba, donde todos los trabajadores en ingenios eran masculinos.

\section{CUADRO 2}

Características de la muestra

\begin{tabular}{|c|c|c|c|c|}
\hline Sector económico & $\begin{array}{l}\text { Proporción } \\
\text { de la muestra }\end{array}$ & $\begin{array}{l}\text { Proporción } \\
\text { capacitados }\end{array}$ & $\begin{array}{l}\text { Proporción } \\
\text { sanos }\end{array}$ & $\begin{array}{c}\text { Proporción } \\
\text { mujeres }\end{array}$ \\
\hline Urbano & 11,8 & 31,4 & 88,8 & 48,7 \\
\hline Estancia/Hacienda . & 3,5 & 2,9 & 77,9 & 43,4 \\
\hline Mixto .............. & 28,4 & 21,8 & 88,2 & 50,7 \\
\hline Ingenio & 35,7 & 16,9 & 81,7 & 42,8 \\
\hline Viña $\ldots .$. & 19,8 & 11,3 & 71,0 & 45,9 \\
\hline Cacao $\ldots \ldots \ldots \ldots \ldots \ldots \ldots \ldots \ldots \ldots$ & 0,8 & 25,0 & 90,0 & 55,0 \\
\hline Total & 100,0 & 17,7 & 82,5 & 46,5 \\
\hline
\end{tabular}

* Varones de 18 años y mayores.

Casi todos los esclavos aparecen en los inventarios con precios positivos. Ello se debe a que la población activa comprendía a la mayor parte de los individuos. Las mujeres normalmente trabajaban en el campo con los hombres: en los ingenios formaban cuadrillas que seguian a los cortadores de caña masculinos haciendo tareas más livianas, como recoger y cargar las cañas y el bagazo; en los viñedos y haciendas de cacao también trabajaban en la cosecha y en el resto de las tareas. En las ciudades una parte importante se desempeñaba en tareas domésticas. Los esclavos viejos y los niños (a partir de los siete u ocho años) también eran útiles, llevando el bagazo seco a las casas de calderas, limpiando los campos de malas hierbas, o cuidando a niños más pequeños.

Del total de la muestra la mayor parte de los individuos (72\%) aparecen como negros criollos, es decir, nacidos en América. Los mulatos (cruza de blan- 
co y negra) representaban el $9 \%$ del total, y los zambos (cruza de indio y negra) el $4,4 \%$. El resto $(14,6 \%)$ procedía de África, probablemente ya aclimatado en su gran mayoria. De una parte de este último grupo se conoce su grupo étnico o región de origen: los Carabali y los Congo representan cada uno $16,4 \%$ de los africanos, los Mandinga 4,8\% y los Mina 3,7\%. Otros que aparecen mencionados en cantidades ínfimas son los Lucumi, Araxa, Angola y Chinos (de Filipinas).

En la muestra los esclavos capacitados aparecen claramente distinguidos del resto sin capacitar ${ }^{8}$. Este era un grupo privilegiado que recibia prebendas y gratificaciones, como pagos monetarios, terrenos para cultivos de usufructo propio, ropa y comida especial, y el uso de caballos para movilizarse. En su inmensa mayoría eran varones; las mujeres no recibirian capacitación, tanto por discriminación como por su menor fuerza y la posibilidad de quedar embarazadas. Entre los esclavos capacitados destacan en primer lugar los contramayorales, también denominados mandadores, que servian de intermediarios entre los administradores y el resto de los negros. Debían coordinar los trabajos del campo, especialmente el de cuadrillas, pudiendo castigar a los que no se esforzaban; además se ocupaban de controlar la distribución de comida. Rendian cuentas, al final de cada día, de las tareas realizadas 9 .

En los centros urbanos (y en las unidades económicas mixtas) aparece un grupo muy variado de trabajadores calificados. En la ciudad de Buenos Aires en 1778 , por ejemplo, los negros y mulatos representaban el $17 \%$ de los artesanos, aunque la probabilidad de un blanco de estar en esa categoría era más del doble que la de alguien de color ${ }^{10}$. En general estos esclavos se encontraban divididos en los tres grados usuales, aprendiz, oficial y maestro, en este último caso con derecho teórico de poseer tienda (lamentablemente en la muestra esta diferenciación no se menciona siempre y no ha podido utilizarse). Los maestros llevaban con ellos al aprendiz, al que le iban enseñando el oficio, en un proceso que se ha descrito como largo ${ }^{11}$. Muchos esclavos urbanos, artesanos o jornaleros, trabajaban en el mercado libre ofreciendo su trabajo; de los montos percibidos por sus servicios debian entregar a sus propietarios una suma fija o proporcional. Tenían relativa libertad y en casos no vivían con sus dueños ${ }^{12}$. Del total de artesanos, una parte importante se dedicaba a la cons-

8 Se ha clasificado como esclavos calificados a aquellas profesiones que aparecen bajo la categoria «craftmen», en Friedman (1992), p. 76.

9 Von Webeser (1986), p. 158; Chevalier (1950), p. 63; Macera (1966), pp. 41-42, 60, 62; Tomich (1990), pp. 240-244.

10 Kossok (1959), p. 115.

11 Chevalier (1950), p. 186; Tomich (1990), p. 225.

12 Saguier (1989). 
trucción y actividades relacionadas: éstos eran los herreros, carpinteros y albañiles. Otra parte incluía a barberos, zapateros, sastres, lomilleros, curtidores, sombrereros y molineros. Algunas especializaciones exigían una mayor capacitación, como la de relojero, músico y escultor.

En los ingenios azucareros un grupo capacitado lo constituían, además de herreros y carpinteros, los esclavos que trabajaban en el procesamiento de la caña y elaboración del azúcar. En primer lugar estaban los caldereros y tacheros, que procesaban el jugo de caña, evaporando el agua en calderas y tachas, con la ayuda de lejias que estimulaban la purificación. Este proceso era muy delicado y un error de cálculo podía arruinar el producto: habilidad especial requeria el determinar el punto de cocción, momento exacto que debía quitarse el jarabe del fuego, para producir una adecuada cristalización. A éstos seguía el purgador, que depositaba el líquido en hormas para que perdiera el resto de humedad. Más tarde se quitaba el azúcar de estos depósitos y lo dividía según su calidad (la peor, más negra, estaba en el fondo). Todo este proceso era coordinado por el maestro de azúcar, que supervisaba todas las tareas y debía tener un conocimiento de la calidad de la caña, que afectaba el grado de cocción que debía recibir. En los viñedos, por su parte, sólo aparecen con una capacidad específica los botijeros y pelleros, a cargo de hacer los envases de barro o cuero, respectivamente, para contener las bebidas alcohólicas producidas. Al parecer otras actividades de los viñedos, como poda, cosecha y pisado, no requerian un entrenamiento digno de notar.

En la muestra un 7,9\% aparece como capacitado, casi todos varones. Dentro del sexo masculino la proporción de los adultos (mayores de 17 años) con alguna capacidad es de $17,4 \%$. Si se distingue por actividad (véase cuadro A), la capacitación es más elevada en el sector urbano, que contaba con un número importante de artesanos y mandadores $(31,4 \%)$. El sector mixto y los ingenios también presentaban una cantidad de trabajadores calificados, $21,8 \%$ y $16,9 \%$, respectivamente. Menor peso tendrian en los viñedos y aún menor en haciendas y estancias. Las cifras indican (cuadro 3) que la proporción de esclavos capacitados aumenta con la edad, hasta la década del cincuenta, luego de la cual cae. Este fenómeno sería mucho más marcado para los establecimientos de tipo plantación (ingenios y viñedos), donde el coste de oportunidad de entrenar a un joven fuerte sería elevado, por lo que se le seleccionaría a una edad más avanzada. En el sector urbano y mixto la capacitación se iniciaria a una edad más temprana, quizá porque no existía esa alternativa. Si se compara la estructura de edad de mandadores y artesanos los primeros aparecen con más frecuencia a edades más tardías que los segundos, pues la madurez parece haber sido un requisito importante a la hora de lograr imponer su autoridad. 
Una instrucción para haciendas jesuitas mexicanas determinaba que entre las cualidades de un mandador estaba el ser «un esclavo fiel, de buen juicio y madura edad» ${ }^{13}$. Este requisito no sería imprescindible en el caso de los artesanos.

\section{CUADRO 3}

Esclavos capacitados

\begin{tabular}{|c|c|c|c|c|c|c|c|c|}
\hline & \multicolumn{3}{|c|}{$\begin{array}{l}\text { Proporción de esclavos } \\
\text { capacitados en cada coborte }\end{array}$} & \multicolumn{5}{|c|}{$\begin{array}{c}\text { Proporción de esclavos capacitados } \\
\text { sobre total de esclavos }\end{array}$} \\
\hline & $\begin{array}{l}\text { Manda- } \\
\text { dores }\end{array}$ & $\begin{array}{c}\text { Artesa- } \\
\text { nos }\end{array}$ & Total & $\begin{array}{l}\text { Manda- } \\
\text { dores }\end{array}$ & $\begin{array}{c}\text { Artesa- } \\
\text { nos }\end{array}$ & Total & $\begin{array}{l}\text { Ingenios } \\
y \text { Viñas }\end{array}$ & $\begin{array}{l}\text { Urbanos } \\
y \text { Mixtos }\end{array}$ \\
\hline $18-27$ & & 22,9 & 20,1 & & 15,2 & 15,2 & 11,9 & 21,8 \\
\hline $28-37$ & 11,1 & 26,0 & 24,2 & 0,9 & 15,6 & 16,5 & 10,9 & 26,4 \\
\hline $38-47 \ldots \ldots \ldots \ldots$ & 22,2 & 26,0 & 25,5 & 2,4 & 20,2 & 22,6 & 19,8 & 34,1 \\
\hline 48.57 & 38,9 & 14,5 & 17,4 & 6,8 & 18,4 & 25,2 & 25,3 & 20,8 \\
\hline $58-67 \ldots \ldots \ldots$ & 11,1 & 9,2 & 9,4 & 2,1 & 12,4 & 14,4 & 11,1 & 28,6 \\
\hline Mayores de 67 & 16,7 & 1,5 & 3,4 & 5,3 & 3,5 & 8,8 & 9,8 & 7,1 \\
\hline Total * & 100,0 & 100,0 & 100,0 & 2,1 & 15,6 & 17,7 & 15,1 & 24,9 \\
\hline
\end{tabular}

* Varones de 18 años y mayores.

Del total de esclavos, el 17,5\% aparece con algún problema de salud. Esta proporción es muy similar a la encontrada por $\mathrm{D}$. Chandler para un conjunto de esclavos de Colombia en el siglo xvill que más que triplica en tamaño a la aquí analizada; alli el porcentaje con algún defecto es de $15,3 \%{ }^{14}$.

En la muestra el padecimiento más común son defectos musculoesqueletales y traumatológicos, como hernias, quebraduras y amputaciones, que representan el $39 \%$ de los no sanos. Con enfermedades de la piel hay un $6,7 \%$, problemas genitourinarios $8,7 \%$, enfermedades de la vista $6,3 \%$, gastrointestinales $4,8 \%$ y pulmonares y respiratorios $8,2 \%$. El resto $(26,3 \%)$ incluye otros padecimientos no detallados, además de afecciones otorrinolaringológicas, neuropsiquiátricas y cardiovasculares. Distinguiendo por actividades, la población más sana es la urbana y mixta, con un 11 a $12 \%$ de las población afectada. En los

13 Chevalier (1950), p. 63.

14 Chandler (1981). De Chandler hemos tomado la tipología de padecimientos. Su muestra se subdivide en esclavos jesuitas (casi la misma cantidad de esclavos que utiliza este trabajo), que presenta una proporción de $20,8 \%$. Véase también Chandler (1982). 
ingenios sube al $18,3 \%$; en estancias y haciendas, al $22,1 \%$, y en los viñedos al $29 \%$. Exceptuando las zonas urbanas, en que los diversos padecimientos están muy distribuidos, en todos los demás los problemas musculoesqueletales son los predominantes, y van del $37 \%$ al $47 \%$ del total, proporción que no puede sorprender dado los accidentes y el desgaste producidos por el rudo trabajo de campo. En ingenios y viñedos destacan también los problemas genitourinarios (en gran parte enfermedades venéreas), probablemente debido a promiscuidad sexual presente en grandes aglomeraciones de individuos. En los viñedos también deben destacarse los problemas pulmonares.

$\mathrm{La}$ incidencia de padecimientos respecto a edad, sexo y capacitación se presenta en el cuadro 4. Como es de esperar, el estado de salud y físico se deteriora con la edad, aunque se estabiliza luego de los 50 años. Las mujeres aparecen con un mejor estado de salud que los varones, especialmente luego de los 40 años. Por otra parte, los esclavos varones capacitados son más sanos; ello puede deberse a que evitaban el trabajo de campo con sus efectos negativos. Alternativamente, podrían haber sido seleccionados entre los que presentaban buena salud.

\section{CUADRO 4}

Proporción de esclavos sanos

\begin{tabular}{|c|c|c|c|c|c|}
\hline \multirow[b]{2}{*}{ Edad } & \multicolumn{3}{|c|}{ Total } & \multicolumn{2}{|c|}{$\begin{array}{c}\text { Varones de } 17 \text { años } \\
\text { y mayores }\end{array}$} \\
\hline & Varones & Mujeres & Total & Capacitados & $\begin{array}{c}\text { No } \\
\text { capacitados }\end{array}$ \\
\hline Menores de 18 & 95,0 & 96,2 & 95,7 & & \\
\hline $18.27 \ldots \ldots \ldots$ & 88,4 & 94,8 & 91,7 & 96,8 & 86,8 \\
\hline $28-37$ & 76,6 & 77,3 & 76,9 & 84,6 & 74,9 \\
\hline 38.47 & 63,1 & 57,4 & 61,0 & 69,2 & 61,2 \\
\hline 48.57 & 59,2 & 65,1 & 61,4 & 74,1 & 54,5 \\
\hline $58-67$ & 60,8 & 63,4 & 61,6 & 64,3 & 60,2 \\
\hline Mayores de 67 & 61,4 & 75,8 & 66,7 & 80,0 & 60,4 \\
\hline Total & 79,5 & 85,8 & 82,5 & 79,3 & 70,1 \\
\hline
\end{tabular}

Problemas de conducta o vicio sólo se destacan en la población de Cuba, donde un $37 \%$ de los esclavos aparece con algún problema de comportamiento. Entre los defectuosos un $61 \%$ era propenso a escaparse, un $22 \%$ alcohólico 
y un $16 \%$ con otros defectos, como el ser ladrón. La característica diferencial de los esclavos cubanos es que eran las únicas plantaciones en que predominaba absolutamente el sexo masculino. Este factor pone en evidencia el papel socializador de la familia, pues en el resto de las unidades económicas estudiadas los sexos están muy balanceados.

\section{LOS DETERMINANTES DEL PRECIO}

Para analizar los determinantes del precio de los esclavos se estiman regresiones que exploran las relaciones estadísticas entre los precios y las características observables de los esclavos en la muestra.

El cuadro 5 contiene diversas estimaciones de ecuaciones de precios. En todos los casos la variable dependiente es el logaritmo del precio. Las variables independientes recogen, en primer lugar, medidas del capital humano de los individuos: edad, capacitación y estado de salud. Con estas variables se pueden estimar ecuaciones similares a las modernas ecuaciones de ingresos de, aunque en este caso la variable a explicar refleje el valor presente descontado de la corriente de rentas que se esperaba generara un esclavo ${ }^{15}$. Las estimaciones incluyen otro conjunto de regresores que reflejan el sexo y la raza de los individuos, para analizar si estas características se asociaban a diferencias de precios. Por último, las ecuaciones recogen la actividad económica en la que se desempeñaban los esclavos.

En el cuadro 5 aparecen, en primer lugar, estimaciones para el conjunto de la muestra. Además de la edad, que presenta una relación cóncava con el precio, la capacitación de los individuos aparece como un claro determinante de su valor, elevándolo aproximadamente un $25 \%$. Si un esclavo se encuentra sano, su precio aumenta como promedio un $66 \%$. Las mujeres en la muestra tienen un precio inferior como media un $7,5 \%$ al de los hombres. Por razas, los africanos se valoran un $20 \%$ más que los criollos; mientras que los mulatos presentan precios inferiores en un $10 \%$ a los de los criollos. Sin embargo, al desagregar los datos por actividades productivas se descubre que estos diferenciales no se producen en todas las actividades. Así, por ejemplo, solamente en las plantaciones de azúcar aparecen diferenciales significativos entre individuos de distintas razas. Las mujeres tienen un precio significativamente inferior al de los hombres únicamente en los viñedos.

15 Mincer (1974). 


\section{CUADRO 5}

Ecuaciones de precio por sector económico

\begin{tabular}{|c|c|c|c|c|c|}
\hline & Todos & Urbano-Mixto & Ingenio & Viña & Hacienda \\
\hline Constante & $3,596(58)$ & $3,383(42)$ & $4,105(38)$ & $4,359(34)$ & $4,231\{35\}$ \\
\hline Edad......... & $0,087(36)$ & $0,076(25)$ & $0,088(19)$ & $0,100(17)$ & $0,064(12)$ \\
\hline $\operatorname{Edad}^{2} \ldots \ldots \ldots \ldots$ & $-0,153(46)$ & $-0,133(29)$ & $-0,155(25)$ & $-0,180(24)$ & $-0,105(14)$ \\
\hline Capacitación ...... & $0,259(4)$ & $0,244(3,2)$ & $0,216(2)$ & $0,493(3)$ & $0,598(2,3)$ \\
\hline Salud $\ldots \ldots \ldots \ldots$ & $0,661(16)$ & $0,913(14)$ & $0,754(10)$ & $0,365(4)$ & $0,181(2,3)$ \\
\hline Mujer ........... & $-0,075(2,4)$ & $-0,013(0,3)$ & $-0,013(0,2)$ & $-0,274(4)$ & $-0,084(1,5)$ \\
\hline Zambo .... & $-0,047(0,6)$ & $-0,032(0,4)$ & $0,018(0,1)$ & $0,238(0,6)$ & \\
\hline Mulato ........... & $-0,109(2,4)$ & $-0,050(1,1)$ & $-0,524(4)$ & $-0,144(0,2)$ & $0,023(0,3)$ \\
\hline Africano $\ldots \ldots \ldots$ & $-0,203(3,4)$ & $-0,156(0,2)$ & $0,271(3)$ & $0,070(0,4)$ & \\
\hline Ingenio & $0,599(16)$ & & & & \\
\hline Viña & $0,505(12)$ & & & & \\
\hline Hacienda & $0,132(1,6)$ & & & & \\
\hline$N$ & 2.347 & 951 & 845 & 468 & 83 \\
\hline $\mathrm{F}$ & 290,1 & 152,6 & 123,1 & 104,3 & 37,8 \\
\hline $\mathrm{R}^{2}$ & 0,57 & 0,56 & 0.53 & 0,64 & 0,73 \\
\hline Edad Pmax. ....... & 28,4 & 28,6 & 28,4 & 27,7 & 30,4 \\
\hline
\end{tabular}

t estadistico entre paréntesis.

Con el objetivo de profundizar en el análisis de las diferentes estructuras de precios, en las cuatro últimas columnas del cuadro 5 se estiman ecuaciones separadas para las distintas actividades económicas. Un test de Chow acepta que se impongan ecuaciones de precios idénticas en el sector urbano y el mixto. Sin embargo, el mismo contraste estadístico rechaza la homogeneidad de las ecuaciones de precios entre el sector urbano-mixto, los ingenios, los viñedos y las haciendas, por lo que se debe concluir que la valoración de las características de los esclavos era diferente en las distintas actividades económicas aquí consideradas. Cabe preguntarse si estas diferencias se debian a la existencia de diferentes mercados por zonas geográficas y actividades. La especificidad del capital humano de los esclavos capacitados puede también ayudar a explicar su diferente valoración en las distintas actividades productivas.

A partir de los datos del cuadro 5 cabe concluir que el valor de la capacitación parece ser mayor en las actividades donde los esclavos calificados eran más escasos, es decir, en viñedos y haciendas. Por el contrario, en estas actividades se observa que el premio correspondiente a un buen estado de 
salud es menor que en los ingenios y en el sector urbano y mixto. En conjunto, las dos características de capital humano consideradas, capacitación y salud, elevan el precio de un esclavo aproximadamente un $110 \%$ en el sector urbano-mixto, un $95 \%$ en los ingenios, un $90 \%$ en las haciendas y un $80 \%$ en los viñedos.

Se comprueba que las estimaciones del cuadro 5 corresponden a edades de precio máximo prácticamente idénticas en las distintas actividades, oscilando entre 27,7 y 28,6 . Unicamente para las haciendas se obtiene un precio máximo diferente, a los 30 años, pero el reducido tamaño de la muestra resta importancia a este resultado. En el gráfico 1 se representan los perfiles

\section{GRÁFICO 1}

\section{Perfiles de precios de esclavos}

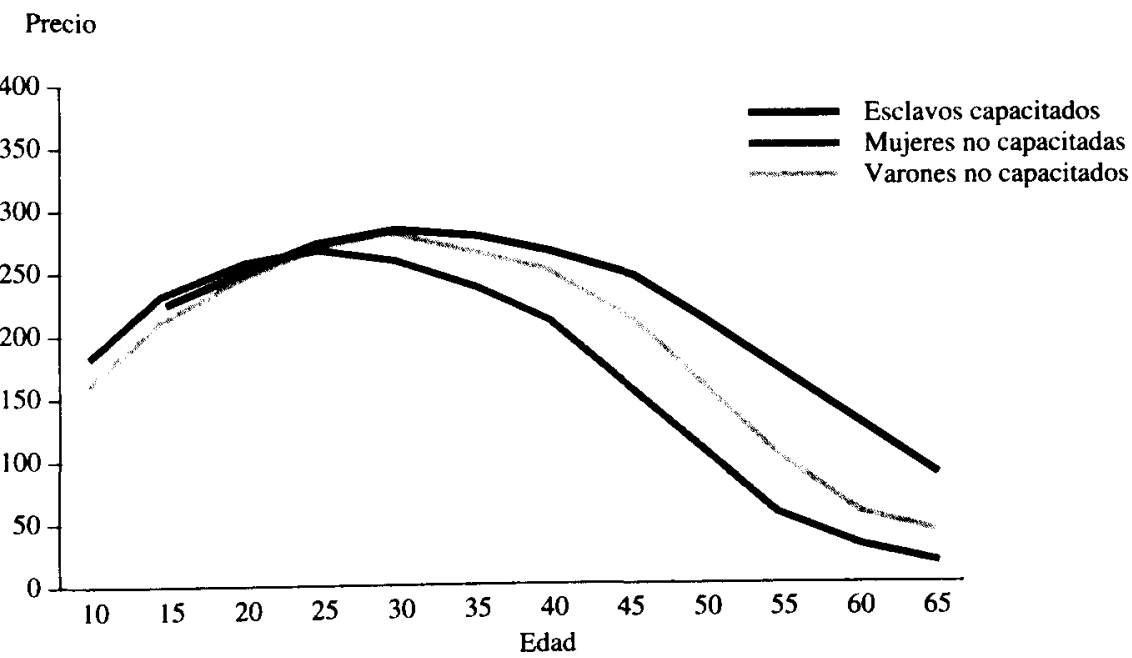

precio-edad estimados a partir de ecuaciones semilogaritmicas para tres grupos de esclavos: hombres y mujeres sin capacitar, y esclavos capacitados ${ }^{16}$. Se observa que a los 10 años las mujeres tenian precios superiores a los de los hombres en un $11,6 \%$, mientras que a los 20 años este diferencial sexual se reduce

16 Casi todos ellos varones. 
al $2 \%$, para hacerse negativo posteriormente. A los 35 años el diferencial a favor de los varones es de $17 \%$ y a los 50 de $52 \%$.

\section{CONCLUSIONES}

Las características de los esclavos hispanoamericanos presentados en este trabajo tienden a confirmar los resultados obtenidos en estudios sobre otras regiones y épocas, mostrando así que la esclavitud ha tenido una estructura relativamente estable, hecho basado en la similitud de las cualidades de las personas, que se reflejaría en su productividad y por ende en sus precios ${ }^{17}$. Las coincidencias incluyen: a) la concavidad de los perfiles edad-precio; b) que el precio de las mujeres llegue a un máximo a una edad más temprana que para los varones; c) que el varón tenga un precio medio superior al de la mujer, invirtiéndose esta relación para los adolescentes; d) que los artesanos aparezcan a edades más tempranas que los mandadores, e) que la mujer presente en general un mejor estado de salud que el varón, y los capacitados que los no capacitados.

\section{FUENTES}

\section{La muestra}

Las tasaciones de las propiedades jesuitas que se realizaron luego de la expulsión de la orden se encuentran fundamentalmente concentradas en el Archivo Histórico Nacional de Chile (AHNCh) y en el Archivo Histórico Nacional de Madrid (AHNM). A continuación se indica la localización de los documentos utilizados en este trabajo:

Montevideo: Molino y Taona, Residencia, Rancheria, Estancia de la Calera, Estancia de Pando (AHNCh, Jesuitas (=J), Libro 146); Santa Fe: Haciendas del Colegio (AHNCh, J, Libro 156); Buenos Aires: Colegio de Belén, Rancheria, Estancia de las Vacas, Chacarita, Estancia de las Conchas, Estancia de la Magdalena (AHNCh, J, Libro 149); Mendoza: Hacienda de la Cañada (AHNCh, J, Libro 156); Santiago del Estero: Rancheria, Hacienda de San Ignacio (AHNCh, J, Libro 151); Salta (AHNCh, J, Libro 149); Corrientes: Chacarita de la Viña, Rancheria (AHNM, J, Legajo 96-1); La Habana: Ingenio San Juan Bautista de Póveda, Ingenio Nuestra Señora de Aranzazi, Ingenio de

17 Sobre las similitudes con esclavos de otras regiones y épocas, véase: Craton (1975); Fogel (1989); Fogel y Engerman (1981); Friedman (1992); Friedman y Manning (1992); Friedman, Manning y Fogel (1992); Geggus (1978); Kotlikoff (1992); Manning (1992); De Mello (1992); Moreno Fraginals et al., (1983); Olson (1992). 
Barrutia (AHNM, J, Legajo 125-2); Guatemala: Ingenio de Amatitlán, Hacienda de los Canales (AHNM, J, Legajo 85-1); Caracas: Hacienda del Valle del Guatiré, Hacienda de San Ignacio (AHNM, J, Legajo 126-2), Hacienda del Valle de Cancagua (AHNM, J, Legajo 128); Ica: Hacienda de San Jerónimo (AHNCh, J, Libro 348), Hacienda San José de Nazca (AHNCh, J, Libro 459); Lima: Hacienda de la Huaca (AHNCh, J, Libro 459); Pisco: Hacienda de Santa Rosa de Caucato (AHNCh, J, Libro 459); Trujillo: Hacienda de Tumán (AHNCh, J, Libro 459); La Serena: Hacienda y Chacra (AHNCh, J, Libro 5).

\section{Bibliografía}

BAUER, A. J. (1990): "Christian Servitude. Slave management in colonial Spanish America», en Mats Lundhal y Thommy Svensson, eds., Agrarian Society in History, Londres, pp. 89-107.

Bergad, L. W. (1987): «Slave prices in Cuba, 1840-1875», Hispanic American Historical Review 67:4, pp. 631-653.

Berger, M., y Leight, P. (1989): «Schooling, Self-Selection and Health», Journal of Human Resources xxiv: 3, pp. $433-455$.

Chandler, D. (1981): Health and Slavery in Colonial Colombia, New York.

Chandler, D. (1982): «The Jesuits and Slavery in Colombia», in Gaetano Massa, ed. Paesi Mediterranei e America Latina, Roma, pp. 286-316.

Chevalier, F., ed. (1950): Instrucción a los Hermanos Jesuitas Administradores de Haciendas, México.

Craton, M. (1975): «Jamaican Slavery», en Engerman y Genovese (1975), pp. 249-284.

Engerman, S. L., y Genovese, E., eds. (1975): Race and Slavery in the Western Hemisphese: Quantitative Studies, Stanford.

FrIEDMAN, G. (1992): «Sources of Data on Slave Occupations: Their Uses and Limitations», en Fogel, Galantine y Manning (1992), pp. 69.77.

Friedman, G., y Manning, R. L. (1992): «The Rent and Hire of Slaves», en Fogel, Galantine y Manning (1992), pp. 77-78.

Friedman, G.; ManNing, R. L., y Fogel, R. W. (1992): «Labor Force Participation and the Life Cycles in Slave Occupations», en Fogel, Galantine y Manning (1992), pp. 140-150.

Fogel, R. W. (1989): Without Consent or Contract. The Rise and Fall of American Slavery, New York.

Fogel, R. W., y Engerman, S. (1981): Tiempo en la Cruz. La economía esclavista en los Estados Unidos, Madrid.

Fogel, R. W., y ENGERMAn, S. (1974): Time in the Cross: Evidence and Methods - A supplement, Londres.

Fogel, R. W., y Engerman, S. (1992): Without Consent or Contract. The Rise and Fall of American Slavery. Markets and Production. Tecbnical papers vol. 1, New York.

Fogel, R. W.; Galantine, R. A., y Manning, R. L. eds. (1992): Without Consent or Contract. The Rise and Fall of American Slavery. Evidence and Metbods, New York.

Galenson, D. W. (1986): Traders, planters and slaves. Market bebavior in early English America, New York. 
Geggus, D. P. (1978): «The Slaves of British-Occupied Saint Domingue: an analysis of the workforces of 197 absentee plantations, 1796-1797», Caribbean Studies 18:1 and 2 (April-July), pp. 5-41.

Grossman, M. (1975): «The Correlation between Health and Schooling», en Nestor Terleckyj ed. Household Production and Consumption, New York.

Higman, B. W. (1976): Slave Population and Economy in Jamaica 1807-1834, Cambridge.

KAPSOLI, W. (1975): Sublevaciones de Esclavos en el Perú s. XVIII, Lima.

KL.EIN, H. (1986): La Esclavitud Africana en América latina y el Caribe, Madrid.

Kossok, M. (1959): El Virreynato del Río de la Plata. Su estructura económico-social, Buenos Aires.

Kotlikoff, L. (1992): «Quantitative Description of the New Orleans Slave Market, 1804 to $1862 \%$, en Fogel y Engerman (1992), pp. 31-53.

MACERA, P. (1966): «Instrucciones para el manejo de las haciendas jesuitas en el Perú (ss. XVII.XVIII)", Nueva Corónica ii: 2, pp. 1-27.

ManNing, R. L. (1992): «The Gang System and the Structure of Slave Employment», en Fogel, Galantine y Manning (1992), pp. 109-119.

MaRCILIO, M. L. (1978): «The price of Slaves in xIxth Century Brazil: a quantitative analysis of the registration of slave sales in Bahia», en Studi in Memoria di Federigo Melis, Nápoles, pp. 83-97.

Marrisey, M. (1989): Slave Women in the New World. Gender Stratification in the Caribbean, Lawrence.

De Mello, P. C.: «Rates of Return on Slave Capital in Brazilian Coffee Plantations, 1871-1881», en Fogel y Engerman (1992), pp. 63-79.

MinCER, J. (1974): Schooling, Experience and Earnings, New York.

Morales Padron, F. (1988): Atlas Histórico Cultural de América latina, 2 vol. Las Palmas.

Moreno Fraginals, M.; Klein, H. y Engerman, S. (1983): «Nivel y estructura del precio de los esclavos en plantaciones de Cuba a mediados del siglo XIX: un estudio comparado», Revista de Historia Económica I: 1, pp. 97-120.

Olson, L. F.: «The Occupational Structure of Southern Plantations during the Late Antebellum Era», en Fogel y Engerman (1992), pp. 137-169.

De Queiros Mattoso, K. M.; Kiein, H., y Engerman, S. L. (1986): "Trends and Patterns in the prices of Manumitted Slaves: Bahia, 1819-1888», Slavery \& Abolition, 7:1 (mayo), pp. 59-67.

Riley, J. D. (1976): Hacendados Jesuitas en México. El Colegio de San Pedro y San Pablo 1685-1767, México.

Rout, L. (1976): The African Experience in Spanish America, Cambridge.

SAGUiER, E. (1989): "La naturaleza estipendaria de la esclavitud urbana colonial. El caso de Buenos Aires en el siglo xviI», Revista Paraguaya de Sociologia, $26: 74$ (eneroabril), pp. 45-54.

Томгсн, D. W. (1990): Slavery in the Circuit of Sugar. Martinique and the World Economy 1830-1848, Londres.

VON WEBESER, G. (1986): «Los esclavos negros en el México Colonial. Las Haciendas de Cuernavaca-Cautla», Jabrbuch für Geschichte Von Staat, Wirtschaft un Gesellschaft Lateinamerikas, pp. 145-171. 